\title{
Using nanosphere lithography for fabrication of a multilayered system of ordered gold nanoparticles
}

\author{
V.I. Styopkin, V.R. Liakhovetskyi, V.I. Rudenko \\ Institute of Physics, NAS of Ukraine, \\ 46, prospect Nauky, 03680 Kyiv, Ukraine, \\ E-mail:vis49@mail.ru; lyakh@iop.kiev.ua; val@iop.kiev.ua
}

\begin{abstract}
New modification of nanosphere lithography has been realized to obtain multilayered systems of ordered gold nanoparticles (NP). NP have been formed using vacuum deposition of 5...60-nm layer of gold on ionic etched multilayered regular coating consisted of several layers of 200-nm polystyrene spheres. Optical study shows that spectra of NP depend on their thickness and may be changed by heat treatment. Increasing the NP thickness within the $5 \ldots 20-\mathrm{nm}$ range leads to a shortwave displacement of the plasmon resonance peak position, while the longwave shift is observed in 20...60-nm range. Heat treatment of NP brings narrowing and displacement of spectral bands, rising the extinction. It has been supposed that variation of the NP shape is the most substantial factor for changes of optical properties in the $5 . .20 \mathrm{~nm}$ thickness region, while electromagnetic coupling between NP in different layers becomes more important for thicknesses larger than $40 \mathrm{~nm}$. Optical properties inherent to the obtained system of NP can be tuned by changing the polystyrene spheres diameter, extent of etching, thickness of gold layer and with the heat treatment. It may be used in design of nanophotonic devices.
\end{abstract}

Keywords: nanosphere lithography, gold nanoparticles, optical properties, heat treatment, ionic etching.

Manuscript received 03.02.17; revised version received 12.04.17; accepted for publication 14.06.17; published online 18.07.17.

\section{Introduction}

Ordered noble metal nanoparticles (NP) are promising materials for photonic materials, SERS, near-field optics, etc. Different lithographic techniques are used to fabricate these nanostructures. The high resolution of NP production can be obtained with electron beam and focused ion beam lithography. However, these methods are serial and expensive, which confines high volume manufacture of NP. An alternative method for production of ordered NP with determined shape is the nanosphere lithography (NSL) [1-3]. NSL is a powerful process to produce various arrays of periodic structures that may be used in the field of nano- and microfabrication. It may be applied in devices of nanophotonics, such as surface enhanced Raman scattering substrates, biosensors, design of ordered nanostructures for ultrahigh density magnetic recording.

In a simplest version, NSL is a two-step process. The one-layer coating of ordered polystyrene spheres (PS) is formed on the substrate. Then, a desired material is deposited on this substrate through holes in the PS layer. In this way, after PS removal one obtains system of ordered NP of this material. But coverage of the substrate with NP is low and amounts 7.2\% [4].

NP in the form of metal film on surface of PS may be used to increase volume of obtained nanostructures $[5,6]$. But the closeness of these NP to each other 
influences strongly on their properties. Moreover, the size of NP is limited by the used sort of PS.

To overcome these disadvantages, in this work NSL method was used to produce ordered NP on the surfaces of etched PS in multilayer coating. Ion etching the PS in coating allows controlling their size and distances between them [7,8]. Besides, in the case of multilayered coating etching PS in the top layer opens those in lower layers and increase a number of the obtained NP. Deposition of metal on the surface of etched PS creates a complex structure of ordered NP that are located in different layers. The aim of the work was to obtain nanostructures, consisted of NP formed on the surface of etched PS in multilayered coatings, and to study there optical properties.

\section{Experiment}

200-nm PS (standard deviation $6 \mathrm{~nm}$ ) were used to fabricate the multilayered coatings. PS were supplied by Sigma-Aldrich (2\% aqueous suspension). The coatings were obtained on glass substrates. Before PS deposition, the substrates were cleaned and hydrophilized. They were maintained in Piranha solution $\left(3: 1 \mathrm{H}_{2} \mathrm{SO}_{4}: \mathrm{H}_{2} \mathrm{O}_{2}\right)$ at $70{ }^{\circ} \mathrm{C}$ for 1 hour. Then, the substrates were rinsed in fresh distilled water and sonicated for $30 \mathrm{~min}$ in 5:1:1 $\mathrm{H}_{2} \mathrm{O}: \mathrm{NH}_{4} \mathrm{OH}: \mathrm{H}_{2} \mathrm{O}_{2}$ solution. Before PS deposition, the substrates were rinsed again in fresh distilled water. Diluted with distilled water to $0.1 \%$ content PS suspension was drop-coated onto substrate and dried at $35^{\circ} \mathrm{C}$. The volume of deposited PS suspension was adjusted to obtain coatings with the thickness of three layers of PS on the substrate. After drying, the coating was formed on the substrate, the main part of which contains three and more layers of PS. Lesser part of substrate was covered with one and two layers of PS.

The PS coatings were etched in JFC-1100 Ion Sputtering Device (JEOL) under the air pressure close to 0.2 Torr. A degree of PS etching depends on the applied anode-cathode voltage, air pressure in the chamber, duration of process, using of ion stream concentrator, etc. The process conditions were adjusted to obtain PS diameters $130 \ldots 150 \mathrm{~nm}$ in the upper layer of coating.

Etched PS were covered with gold by using thermal evaporation in vacuum. The weight thickness of gold layer varied from 5 up to $60 \mathrm{~nm}$. Thus, it was obtained a multilayered system of gold NP on the surface of etched PS. To enhance stability, the NP were thermally treated. 5 -min heat treatment was done in air at $270 \ldots 300^{\circ} \mathrm{C}$. It should be noted that uncovered PS soften at $120 \ldots 140^{\circ} \mathrm{C}$ for $5 \mathrm{~min}$, but the gold coating makes it possible to sufficiently rise the temperature of heat treatment.

Morphology of this multilayer system of gold NP was studied using JSM-35 scanning electron microscope (SEM).

Optical spectra of the NP system were obtained with MDR-6 spectrometer within the range 400 to $800 \mathrm{~nm}$ with a non-polarized incident light beam at room temperature.

\section{Results}

SEM image of a part of the multilayered PS coating is shown in Fig. 1a. Two PS are lacking in the upper layer and PS of the second layer become visible at these places. Scheme of PS packing in such a coating is shown in Fig. 1b.

After ion etching, PS in upper layers reduce and PS of the second and third layers appear. SEM image of the multilayered PS coating after ion etching and deposition of the gold layer are shown in Fig. 2a. PS in the upper layer decrease down to $140 \ldots 150 \mathrm{~nm}$, while the size of visible part of PS in the second layer reduces down to $160 \ldots 170 \mathrm{~nm}$. The scheme of PS packing after etching is shown in Fig. 2b.

Heat treatment reduces the size of PS and NP on the PS, correspondingly. The PS and NP sizes reduction can be observed on SEM image of the system after heat treatment (Fig. 3). This reduction will give an increase of NP thickness.
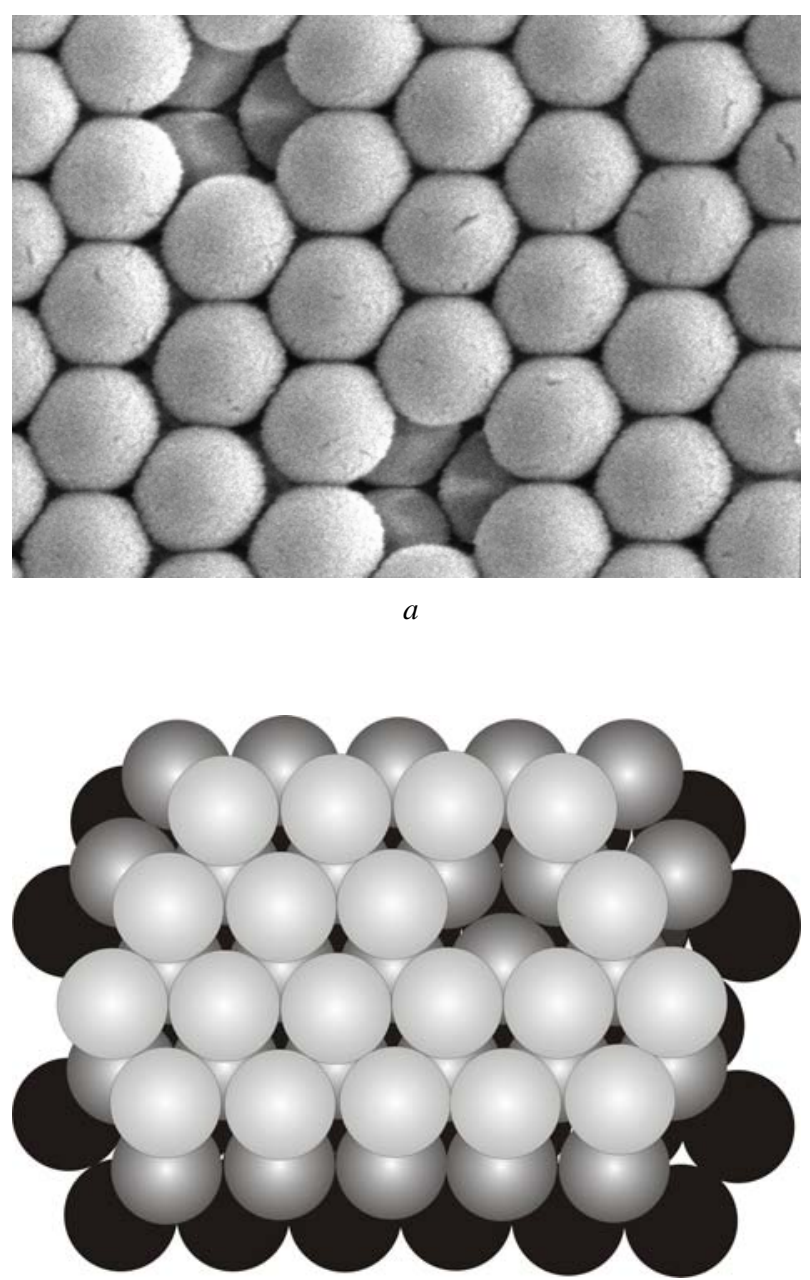

$b$

Fig. 1. SEM image of the multilayered PS coating with defects in the upper layer (a) and the scheme of this coating (b). 


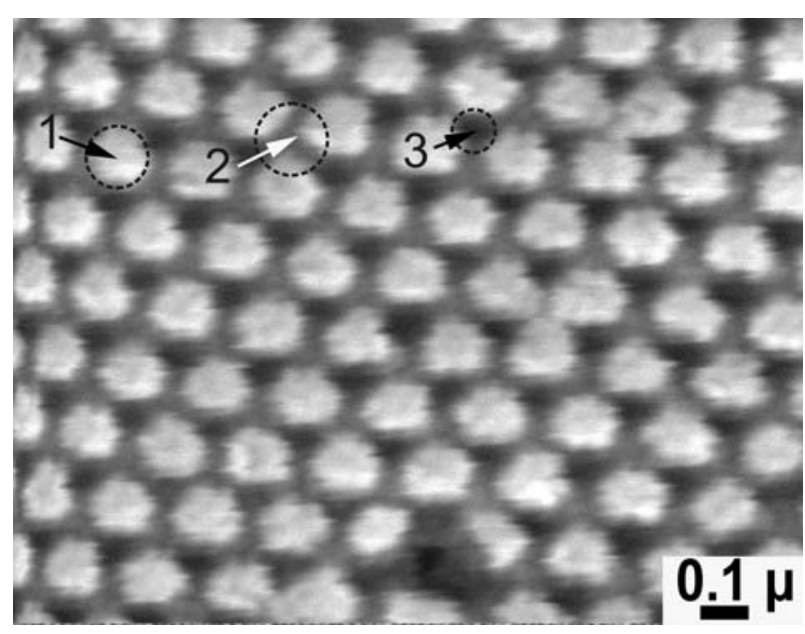

$a$

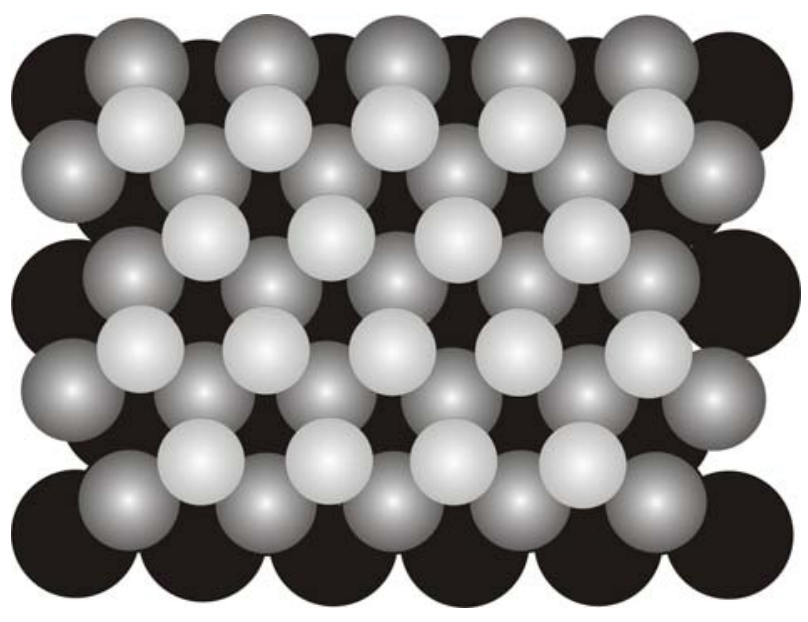

$b$

Fig. 2. SEM image of the multilayered PS coating after ion etching : 1 - PS in the upper layer, 2 - PS in the second layer, 3 - PS in the third layer (a); scheme of PS packing after etching (b).

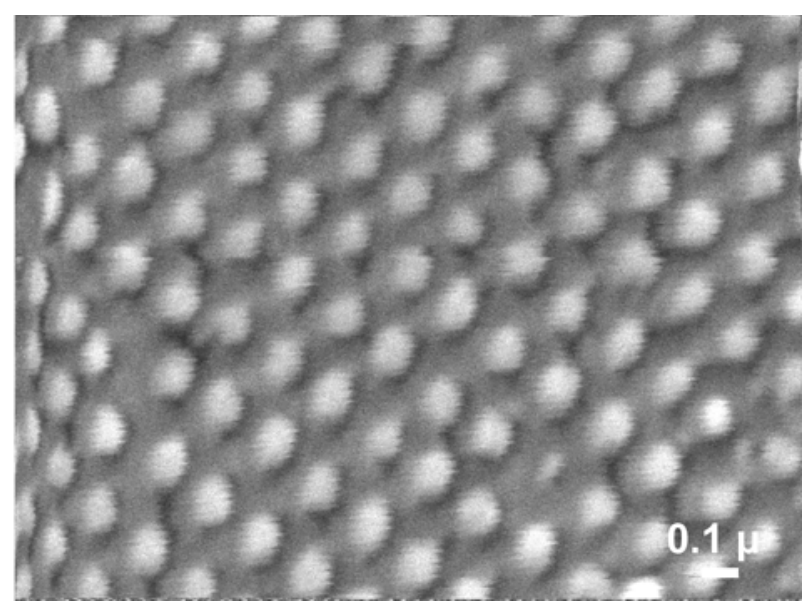

Fig. 3. SEM image of the multilayered NP system after heat treatment.
Optical density spectra of the multilayered system of etched PS after deposition of gold layer of different thickness and the spectrum of the initial PS coating are shown in Fig. 4a. The spectra of gold NP on etched PS reveal broad peaks. The extinction rises with the increasing of gold layer thickness. The position of spectra maximum displaces to the lower wavelengths with thickening the gold layer up to $40 \mathrm{~nm}$. But the resonance peak of $60-\mathrm{nm}$ NP shifts to a longer wavelengths in comparison with that of 40-nm NP. Fig. $4 \mathrm{~b}$ shows that heat treatment of NP leads to narrowing the spectral bands, rising the extinction and displacing the peak position. The value and sign of this displacement depends on the gold layer thickness. As a result, the wavelength of spectra maximum lowers with thickening the gold layer within the range $5 \ldots 20 \mathrm{~nm}$, while within the range $20 \ldots 60 \mathrm{~nm}$ of gold thickness the wavelength rises. Thus, heat treatment gives a different sign of displacement for the spectra in different regions of gold NP thicknesses.

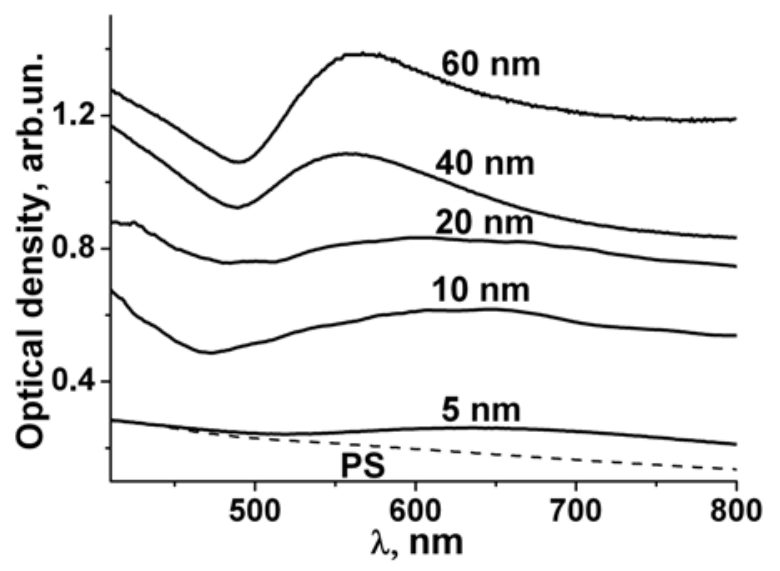

$a$

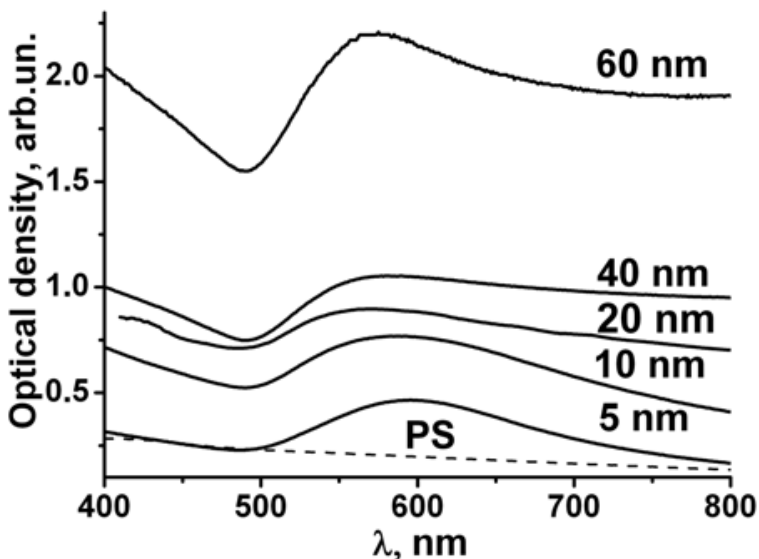

$b$

Fig. 4. Optical density spectra of PS coating and of multilayered system of gold NP with different thicknesses on the etched PS before (a) and after heat treatment (b).

(C) 2017, V. Lashkaryov Institute of Semiconductor Physics, National Academy of Sciences of Ukraine 
Fig. 5 shows the optical density spectra of multilayered system of gold NP with the thickness values 20 and $40 \mathrm{~nm}$ before and after heat treatment. In the case of $20 \mathrm{~nm}$, the thickness position of spectrum maximum changes from 604 to $570 \mathrm{~nm}$ after heat treatment, while for the thickness $40 \mathrm{~nm}$ - from 557 to $579 \mathrm{~nm}$.

\section{Discussion}

Optical spectra of noble metal NP are mainly controlled by localized surface plasmon resonance (LSPR). Characteristics of this resonance depend on the size, shape, properties of substrate. They may also be influenced by electromagnetic coupling between the adjacent NP. In this work, optical properties of the multilayered NP system show good correlation with data of gold NP in other works. The spectra of rectangular gold NP are calculated in [9]. Peak of dipole LSPR is obtained at $545 \mathrm{~nm}$ for the system of NP with 200-nm size, $50-\mathrm{nm}$ thickness and interparticle distance of $200 \mathrm{~nm}$. In our work, NP with 150-nm size and 60-nm thickness show the peak at $575 \mathrm{~nm}$. This 30-nm displacement may be caused by difference in the NP shape and influence of substrate.

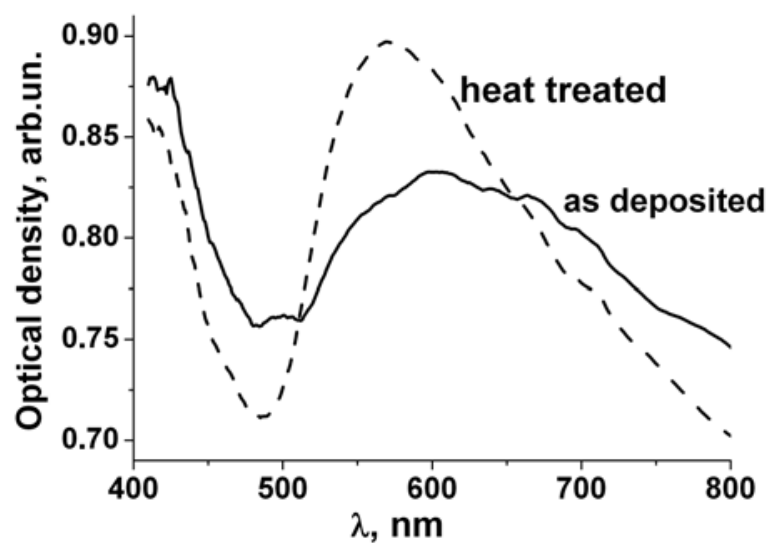

$a$

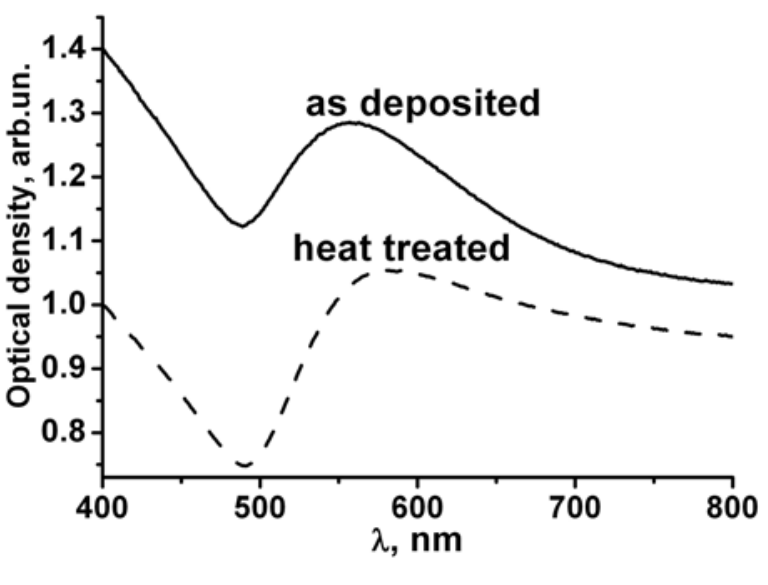

$b$

Fig. 5. Optical density spectra of the multilayered system of gold NP on the etched PS before and after heat treatment with the thickness of gold $20 \mathrm{~nm}$ (a) and $40 \mathrm{~nm}$ (b).
The conditions of NP formation in [10] are similar to those of this work. The gold NP with the thickness $30 \mathrm{~nm}$ were obtained on 170-nm PS on a glass substrate. Resulting NP system shows spectra with the peak at $600 \mathrm{~nm}$. This is also close to the results of our work.

To explain changes in the spectra with variation of NP thickness and after heat treatment, only dipole resonance has been considered in this work. We supposed that optical properties of multilayered NP system mainly depend on the NP thickness and interaction of electromagnetic fields of nearest NP.

In the region of small NP thickness, its variation will not change the interparticle distance of the NP in the same layer. NP of the different layers are placed at quite large distance, and electromagnetic coupling of NP is low. Thus, variation of distance between these NP will not influence sufficiently on their properties, and optical spectra of NP system mainly depend on variation of single NP properties.

Thickening the NP will change their shape. This effect was considered for NP with the shape of spheroids [11] and rods [12]. Such changing is also studied for variation of aspect ratio of rectangular gold NP [9]. Calculations show a long wave shift of peak position for length increase of long side of NP. This displacement is explained by the influence of phase retardation of incident radiation and by electrodynamics boundary effects related with the particle shape. The effect of phase retardation is revealed at relatively large sizes of $\mathrm{NP}$, when the induced polarization is not all in-phase for plane-wave excitation due to the finite wavelength. In our case, NP sizes are about $150 \mathrm{~nm}$, and effect of phase retardation may be observed. But the increase in the NP thickness does not change the NP size in the plane of incident light polarization, and phase retardation cannot cause evident changes in the optical spectra. To a greater extent, it should influence variation of the NP aspect ratio. Thickening the NP increases the ratio of thickness to a size of NP that is in the plane of incident light polarization. Thus, the resonant peak position will shift to the short wave side.

A similar shortwave shift with NP thickening was obtained for resonance in Ag nanodisks [12], where it was shown that such a shift is more significant at a small thickness.

On the other hand, electromagnetic coupling between NP will exhibit sufficient effect at larger NP thickness. This interaction between particle fields involves both very short-distance interactions due to evanescent fields and long-range interactions, mainly due to propagating dipolar fields. It also causes displacement of the resonant peak. The value and sign of this displacement depend on orientation of light polarization relative to interacting NP. Study of electromagnetic coupling of gold NP pairs [13] shows that the decrease in distance between NP leads to a longwave shift of resonance in the case of parallel orientation of light polarization to the long axis of particle pairs. In the case of orthogonal orientation of 
light polarization, the shortwave displacement is observed.

In the considered multilayered system, NP are located in different layers at various distances. So, electromagnetic coupling between NP is very complicated. The scheme of NP arrangement in different layers is shown in Fig. 6 (side view). The schematic charge distribution in NP is also given for the excitation with light having polarization parallel to axis passing the centers of 1 and 2 NP.

Increasing the NP thickness does not change the distance between NP 1 and 2 located in one layer. Distance between NP 3 in the second layer and NP 1 and 2 in upper layer decreases. Thus, thickening the NP will influence mainly on the electromagnetic coupling between NP in different layers.

Fig. 7 shows the scheme of NP arrangement in different layers (top view) and for charge distribution in them under two orientations of light polarization. In the case of light polarization in Fig. 7a, electromagnetic coupling of NP 3 and 4 gives a longwave shift of resonance. Charges in NP 1 and 2 are arranged almost symmetrically relative to charges in NP 3 and coupling between these particles will not change resonance noticeably. In the case of light polarization in Fig. 7b, electromagnetic coupling of NP 3 and 4 leads to a shortwave shift of resonance, while coupling of NP 3 with particles 1 and 2 gives longwave shift. One may guess that interaction of NP 3 with two particles (1 and 2) gives a larger effect than that with one NP (4). Thus, it may be supposed that in our multilayered NP system electromagnetic interaction of NP in different layers will lead to a longwave shift of resonant peak. But this interaction will give noticeable effect on plasmonic resonance only at the small distance between NP. Results of studying the gold NP [13,14] show that for 100-nm NP interaction coupling reveals sufficient effect on optical spectra at the interparticle distance smaller than $50 . .60 \mathrm{~nm}$.

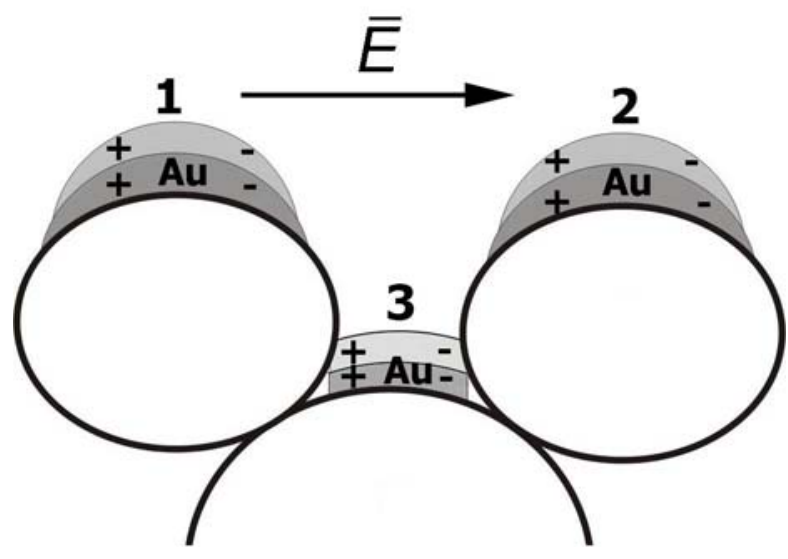

Fig. 6. Scheme of arrangement of NP in different layers (side view) and charge distribution in them under excitation with light.

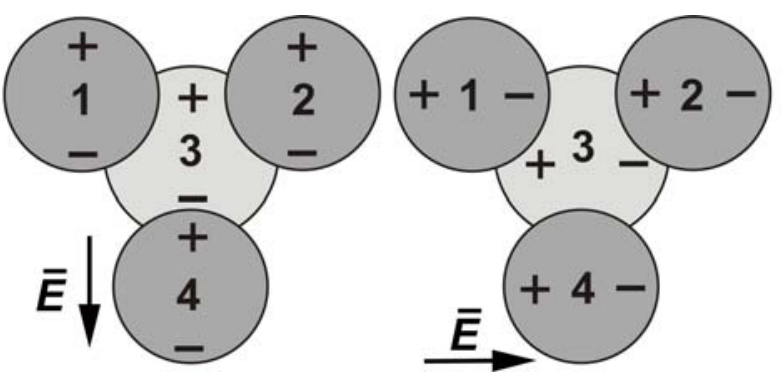

Fig. 7. Scheme of arrangement of NP in different layers (top view) and charge distribution in them under two orientations of light polarization.

From changes in the PS size after etching, it may be supposed that the distance between adjacent NP in different layers amounts about $100 \mathrm{~nm}$. Increasing the $\mathrm{NP}$ thickness up to $30 \ldots 40 \mathrm{~nm}$ will lower the interparticle distance down to $60 \ldots 70 \mathrm{~nm}$. Results of $[14,15]$ give the longwave shift of resonance $5 \ldots 10 \mathrm{~nm}$ for these distances.

Therefore, the resonance spectra will reveal a shortwave shift with the NP thickening mainly due to variation of single NP properties up to $40 \mathrm{~nm}$ thickness. Subsequent rise of the thickness will increase electromagnetic coupling of NP at different layers and lead to a longwave shift of resonance.

The same reasons may explain the changes in spectra after heat treatment. As it was shown earlier, heat treatment reduces the size of PS and NP and increases thickness of NP. The above discussion leads to a supposition that within the range $0 \ldots 40 \mathrm{~nm}$ the thickness increasing after heat treatment will shift resonance to a shortwave part of spectra, while for thicknesses larger $40 \mathrm{~nm}$ - to the longwave part.

\section{Conclusions}

In this paper, we have shown that using NSL in combination with the ionic etching allows to obtain multilayered system of ordered metal NP. Optical spectra of the initial NP system and thermally treated NP were studied. It has been shown that optical properties of the multilayered NP system may be tuned by varying the NP thickness and heat treatment. Thickening the NP shifts LSPR spectra, value and sign of displacement depends on the NP thickness. The heat treatment gives enlarging the NP thickness and change optical properties, too. Considering the influence of NP thickness on spectra has shown two main reasons for resonance modification. Changing the NP shape is the most substantial factor in the thickness range $5 \ldots 20 \mathrm{~nm}$, while the electromagnetic coupling between NP in different layers becomes more important for thicknesses larger than $40 \mathrm{~nm}$.

The proposed system of ordered NP may be used to design nanophotonic devices. 


\section{References}

1. Zhang X., Whitney A.V., Zhao J., Hicks E.M., and Van Duyne R.P. Advances in contemporary nanosphere lithographic techniques. J. Nanosci. Nanotechnol. 2006. 6. P. 1-15.

2. Ye X., Qi L. Two-dimensionally patterned nanostructures based on monolayer colloidal crystals: Controllable fabrication, assembly, and applications. Nano Today. 2011. 6. P. 608-631.

3. Colson P., Henrist C., and Cloots R. Nanosphere lithography: A powerful method for the controlled manufacturing of nanomaterials. J. Nanomater. 2013. 2013. P. 1-19.

4. Haynes C.L. and Van Duyne R.P. Nanosphere lithography: A versatile nanofabrication tool for studies of size-dependent nanoparticle optics. $J$. Phys. Chem. B. 2001. 105. P. 5599-5611.

5. Baia L., Baia M., Popp J., Astilean S. Gold films deposited over regular arrays of polystyrene nanospheres as highly effective SERS substrates from visible to NIR. J. Phys. Chem. B. 2006. 110, No. 47. P. 23982-23986.

6. Fracau C., Canpean V., Gabor M., Petrisor T., Astilean S. Periodically nanostructured noble-metal thin films with enhanced optical properties. $J$. Optoelectron. Adv. Mater. 2008. 10, No. 4. P. 809812.

7. Choi D.-G., Yu H.K., Jang S.G., and Yang S.-M. Colloidal lithographic nanopatterning via reactive ion etching. J. Am. Chem. Soc. 2004. 126. P. 70197025.

8. Zhang Y., Wang X., Wang Y., Liu H., Yang J. Ordered nanostructures array fabricated by nanosphere lithography. J. Alloys and Compounds. 2008. 452. P. 473-477.
9. Atkinson A.L., McMahon J.M., and Schatz G.C. FDTD Studies of Metallic Nanoparticle Systems. In: Self-Organization of Molecular Systems: From Molecules and Clusters to Nanotubes and Proteins. Eds. N. Russo, V.Y. Antonchenko, E.S. Kryachko. NATO Science for Peace and Security, Series A: Chemistry and Biology. Springer Science, Dodrecht, 2009. P. 11-32.

10. Xiaodong Zhou and Nan Zhang, Profile controlled gold nanostructures fabricated by nanosphere lithography for localized surface plasmon resonance. World Academy of Science, Engineering and Technology. 2010. 68. P. 794-800.

11. Kelly K.L., Coronado E., Zhao L., Schatz G.C. The optical properties of metal nanoparticles: The influence of size, shape, and dielectric environment. J. Phys. Chem. B. 2003. 107. P. 668677.

12. Payne E.K., Shuford K.L., Park S., Schatz G.C., and Mirkin C.A. Multipole plasmon resonances in gold nanorods. J. Phys. Chem. B. 2006. 110, No. 5. P. 2150-2154.

13. Henson J., DiMaria J., and Paiella R. Influence of nanoparticle height on plasmonic resonance wavelength and electromagnetic field enhancement in two-dimensional arrays. J. Appl. Phys. 2009. 106. P. 093111.

14. Rechberger W., Hohenau A., Leitner A., Krenn J.R., Lamprecht B., Aussenegg F.R. Optical properties of two interacting gold nanoparticles. Optics Communs. 2003. 220. P. 137-141.

15. Su K.-H., Wei Q.-H., Zhang X., Mock J.J., Smith D.R., Schultz S. Interparticle coupling effects on plasmon resonances of nanogold particles. Nano Lett. 2003. 3. P. 1087-1090. 